\title{
Ectopic fat stores: housekeepers that can overspill into weapons of lean body mass destruction
}

\author{
International Journal of Obesity (2004) 28, S1-S2. doi:10.1038/sj.ijo.0802851
}

\section{Introduction}

Type 2 diabetes and cardiovascular diseases are on track to become the worst killers of the 21st century. How obesity provokes these debilitating diseases appears to reside in the accumulation of lipids outside 'classical' adipose tissue depots, that is, in or around organs and tissues that constitute the lean body mass (skeletal muscle, heart, liver, pancreas, kidneys, blood vessels). This preface to the proceedings of the 2nd Fribourg Obesity Research Conference provides a brief perspective and overview of this conference about 'why and how fat might be stored in the wrong places', and about the metabolic and mechanical consequences of such ectopic fat storage in the pathogenesis of type 2 diabetes and cardiovascular diseases.

With more than 300 millions adults obese and a surge in the prevalence of childhood obesity worldwide (including in Switzerland), the global obesity epidemic - which it is the driving force behind an equally alarming explosion of type 2 diabetes and cardiovascular diseases-has turned into a global 'pathobesity' crisis. This grim picture is further compounded by the latest report from the International Obesity Task Force (IOTF) that more than 1 billion men, women and children are already overweight, and that even a modest gain in body fat can exert a powerful impact on the risks for these chronic diseases among those who are genetically and physiologically vulnerable. Understanding the pathophysiology of obesity is therefore an issue of utmost importance towards developing strategies to cope with this health challenge.

\section{Fat distribution and diseases}

Ever since obesity was introduced into the International Classification of Diseases half a century ago, research towards understanding how excess fat predisposes to chronic diseases has been dominated by concepts centered upon the specific locations at which fat accumulates in the body. In the 1950s, Jean Vague ${ }^{1}$ proposed that excess fat stored on the trunk or android obesity could be metabolically more damaging than fat stored on the limbs (or gynoid obesity). This proposal has been confirmed by a large number of cross-sectional and prospective studies, and it is now recognized that abdominal (or central) obesity is a cardinal feature of the metabolic syndrome, with insulin resistance as a key link between abdominal fat and risks for chronic diseases. Whether specific anatomical compartments in the abdominal region confer greater risk for insulin resistance and its complications is however controversial. Many studies do in fact support the concept of a specific role for intra-abdominal fat accumulation (or visceral obesity) in the link between abdominal obesity and insulin resistance, with the causative mechanism attributed to the release of free fatty acids (FFAs) from visceral fat depots, which by draining into the portal vein would exert adverse effects on hepatic metabolism. However, as Keith Frayn has argued, ${ }^{2}$ there is no clear proof of such a causal link between visceral adiposity and insulin resistance. Both are in fact common correlates of subcutaneous abdominal adipose tissue accumulation, which on the basis of its considerably larger mass than visceral fat could have a greater potential to contribute to insulin resistance through the release of FFAs into the systemic circulation.

While these issues of regional fat adiposity in the pathophysiology of obesity continue to be a subject for debate, the limelight over research towards understanding how obesity leads to chronic diseases has shifted towards the radically novel concept that the pathogenicity of excess fat accumulation lies primarily outside the 'classical' adipose tissue depots. Initiated by Roger Unger in the mid-1990s, ${ }^{3}$ an important causative link between fat distribution and the metabolic syndrome is now thought to reside in the accumulation of lipids in cells that constitute the organs and tissues of the lean body mass - notably in the pancreas, liver, skeletal muscle and heart. As Unger elegantly argues, ${ }^{3}$ the small intracellular reserve of lipids for essential 'housekeeping' functions in nonadipocytes - such as for the maintenance of membrane structure, fluidity and intracellular signalling-is tightly regulated, and if overloaded would lead to cell dysfunction (lipotoxicity) and lipid-induced programmed cell death (lipoapoptosis). In other words, such ectopic lipid reserves can overspill into weapons of lean body mass destruction. ${ }^{4}$

Focus on ectopic fat storage and lipotoxicity This supplement of the International Journal of Obesity reports the proceedings of the 2nd Fribourg Obesity Research Conference (FORC-2003), held in Switzerland, which focused on 
this rapidly evolving topic of ectopic fat storage and lipotoxicity from a perspective of both systemic and molecular (patho)physiology. The theme on 'lipotoxicity' encompasses toxicity that results not only from lipid overloading induced by excess delivery $v s$ oxidation of circulating FFAs but also from lipids synthesized from an overload of glucose by the process of de novo lipogenesis occurring in adipocytes or in nonadipocytes. In fact, in his critical reappraisal of the theory of fat balance in humans, Yves Schutz underscores the fact that, because indirect calorimetry can only track net (rather than total) de novo lipogenesis, the importance of de novo lipogenesis in human obesity and related disorders has most probably been underestimated. To follow-up on the complex relationship between nutrient metabolism and chronic diseases, Eric Ravussin (see Heilbronn et al) first overviews the evidence linking ectopic fat storage to insulin resistance and type 2 diabetes, and then discusses what one might call the 'push and pull' pathophysiological pathways to ectopic fat storage, that is, (i) the failure of adipose cells to proliferate and to buffer lean body mass against excessive exposure to circulating FFAs (the 'push' factor), and (ii) impairments in the capacity of organs/ tissues of the lean body mass to increase fat oxidation (the 'pull' factor) - which could result from alterations in the secretion or sensitivity to adipocyte-secreted factors, such as leptin, adiponectin, resistin and an ever increasing number of adipokines.

The rapid advances in unravelling the molecular mechanisms that underscore these 'push and pull' pathways owe much to the use of animal models. Following an analysis of studies that utilize both transgenic and knockout murine models, Antonio Vidal-Puig (see Lelliott and Vidal-Puig) proposes a series of molecular models to describe mechanisms by which both adipose and nonadipose tissues could predispose to ectopic fat storage and the metabolic syndrome. Abdul Dulloo, from an analysis of studies that integrate data of respiration rate from mouse skeletal muscle in response to leptin and pharmacological interference with intermediary metabolism, proposes that the protective effects of leptin against ectopic fat storage are mediated by substrate cycling between de novo lipogenesis and lipid oxidation, and that this thermogenic effector in skeletal muscle is orchestrated by both PI3 kinase and AMP-activated protein kinase signalling. Jan Kopecky (see Rossmeisl et al), on the basis of his work in mice whose resistance to obesity resides in (genetically engineered) uncoupling of white adipocyte mitochondria, emphasizes the role of cellular ATP and AMP-activated protein kinase in adipose metabolism, and their importance in maintaining the integrity of the adipose tissue as efficient buffer against ectopic fat storage. These presentations about the mechanisms that could protect or predispose to ectopic fat storage were complemented, by Françoise Rohner-Jeanrenaud (see Asensio et al), with an integrative analysis of brain-periphery interactions through which glucocorticoids might influence crosstalks between adipose tissue and skeletal muscle, or between adipose tissue and liver, to provoke insulin resistance and hepatic glucose overproduction, respectively.

A second session was devoted to the pathways from ectopic fat storage to chronic diseases with (i) new insights from Françoise Assimacopoulos-Jeannet into how long-term exposure to circulating FFAs and lipid overloading will eventually lead to lipotoxicity and lipoapoptosis in pancreas and insulin-sensitive tissues - all of which will provoke or exacerbate type 2 diabetes, followed by (ii) an integrative analysis, by Jean-Pierre Montani, of the mechanisms by which fat storage in and around key target organs of cardiovascular control (heart, kidneys and blood vessels) may impair their functions, and hence contribute to the increased prevalence of cardiovascular diseases in obese subjects. A common feature in several of these above-mentioned contributions is the recognition that it may not be the expansion of the lipid stores per se (essentially as triglycerides) that is harmful to the cells of the lean body mass, but rather the overspilling of their fatty acid moieties (eg diglycerides and fatty-acyl CoA). Nowhere is this distinction between 'good' and 'bad' lipid pools more apparent than in comparing the obese and the endurance-trained athlete-both of which have elevated intramyocellular triglycerides but are at opposite ends of the spectrum of insulin sensitivity. As Aaron Russell points out in his analysis of this apparent 'paradox', the breakdown in the relationship between intramyocellular triglycerides and insulin sensitivity by endurance-trained athletes might well be explained by considering the peroxidation status of the intramyocellular triglycerides rather than triglycerides content of the muscle per se.

\section{Conclusions}

In summary, this supplement contains a set of papers that integrates, from a molecular-(patho)physiological perspective, the latest findings and emerging concepts about 'why and how fat might be stored in the wrong places', and discusses both the metabolic and mechanical consequences of such ectopic fat storage in the pathogenesis of type 2 diabetes and cardiovascular diseases.

\author{
AG Dulloo ${ }^{1}$, V Antic ${ }^{1}$ and J-P Montani ${ }^{1}$ \\ ${ }^{1}$ Department of Medicine, Division of Physiology, University of \\ Fribourg, Switzerland
}

\section{References}

1 Vague J. The degree of masculine differentiation of obesities: a factor determining predisposition to diabetes, atherosclerosis, gout and uric calculous disease. Am J Clin Nutr 1956; 4: 20-34.

2 Frayn KN. Visceral fat and insulin resistance-causative or correlative. Br J Nutr 2000; 83 (Suppl 1): S71-S77.

3 Unger RH, Zhou YT, Orci L. Regulation of fatty acid homeostasis in cells: novel role of leptin. Proc Natl Acad Sci USA 1999; 96: 2327-2332.

4 Unger RH. Weapons of lean body mass destruction: the role of ectopic lipids in the metabolic syndrome. Endocrinology 2003; 144: 5159-5165. 